\title{
Quantitative X-ray Microanalysis of Low Atomic Number Elements by SEM/SDD-EDS with NIST DTSA II: Carbides and Nitrides and Oxides, Oh My!
}

\author{
Dale E. Newbury and Nicholas W. M. Ritchie \\ National Institute of Standards and Technology, Gaithersburg, MD 20899-8370
}

Quantitative electron-excited x-ray microanalysis of the low atomic number elements, Be, B, C, N, O, and $\mathrm{F}$ that can only be measured with $\mathrm{X}$-ray peaks whose energies fall below $1 \mathrm{keV}$, has presented a great challenge since the earliest years of the technique [1]. Typically measured with wavelength dispersive spectrometry (WDS) by following the k-ratio protocol, the low energy photon peaks are vulnerable to strong effects on x-ray peak position and shape that depend on chemical binding, as described in detail in the classic series of papers by Bastin and Heiligers [2], who developed a "peak-shape" factor correction to compensate for chemical shifts that affected fixed peak-channel WDS measurements. The spectrum measurement problem is further complicated by peak interferences that often arise from the L-family and M-family x-rays of heavier elements that occur in this energy range. Direct quantitative analysis of light elements by energy dispersive x-ray spectrometry (EDS) has generally been avoided, with the expedient of calculating oxygen by assumed stoichiometry for fully oxidized systems. The analytical situation for low-Z elements has changed with the advent of the silicon drift detector (SDD) with its high throughput, extreme peak stability, and improved resolution and sensitivity for low-energy photons. The high throughput enables high count EDS spectra to be accumulated in modest time, e.g., $10^{7}$ count spectra in $100 \mathrm{~s}$. The resulting stable, high count peak references for multiple linear least squares (MLLS) peak fitting of unknowns and standards enable SEM/SDD-EDS to follow the k-ratio protocol and match WDS precision and accuracy for a vast range of measurement problems, even when severe peak interference occurs [3]. We have extended this work to demonstrate that SEM/SDD-EDS can perform accurate quantitative microanalysis of carbides, nitrides, and oxides, including examples with significant peak interference. The analytical strategy included selection of a beam energy of $10 \mathrm{keV}$ to provide access to the K-shell of the transition elements and L-shell of higher atomic elements; a beam current to restrict detector deadtime to approximately 10 percent to minimize coincidence peaks; and a dose to provide approximately $10^{7}$ integrated counts. EDS spectra were collected with commercial software and NIST DTSA II [4] was used for all spectrum processing (MLLS peak fitting) and matrix corrections. An example of the peak fitting in the low photon energy region ( $\mathrm{N} \mathrm{K}$ and $\mathrm{Cr} \mathrm{L}$ ) is shown in Figure 1, while Tables 1, 2 and 3 list the results (in atom fractions) for various carbides, nitrides, and oxides. The results demonstrate sufficient accuracy to readily distinguish among compounds with small differences in the mass fraction of the low- $\mathrm{Z}$ constituent: $\mathrm{Cr}_{3} \mathrm{C}_{2}, \mathrm{Cr}_{23} \mathrm{C}_{6}$, and $\mathrm{Cr}_{7} \mathrm{C}_{3} ; \mathrm{SiO}_{2}$ and $\mathrm{SiO}$; and $\mathrm{CuO}$ and $\mathrm{Cu}_{2} \mathrm{O}$.

\section{References:}

[1] J. Goldstein et al., Scanning Electron Microscopy and X-ray Microanalysis, $3^{\text {rd }}$ ed. (Springer, New York, 2003) 391.

[2] G. Bastin and H. Heijligers, in Electron Probe Quantitation, K Heinrich and D. Newbury, eds. (Plenum, New York, 1991) 163-175.

[3] N. Ritchie, D. Newbury, and J. Davis, Microsc. Microanal., 18 (2012) 892.

[4] N. Ritchie, DTSA-II available free at: www.cstl.nist.gov/div837/837.02/epq/dtsa2/index.html 
Figure 1 SDD-EDS spectrum of $\mathrm{Cr} 2 \mathrm{~N}$ and residuals after MLLS fitting with NIST DTSA II.
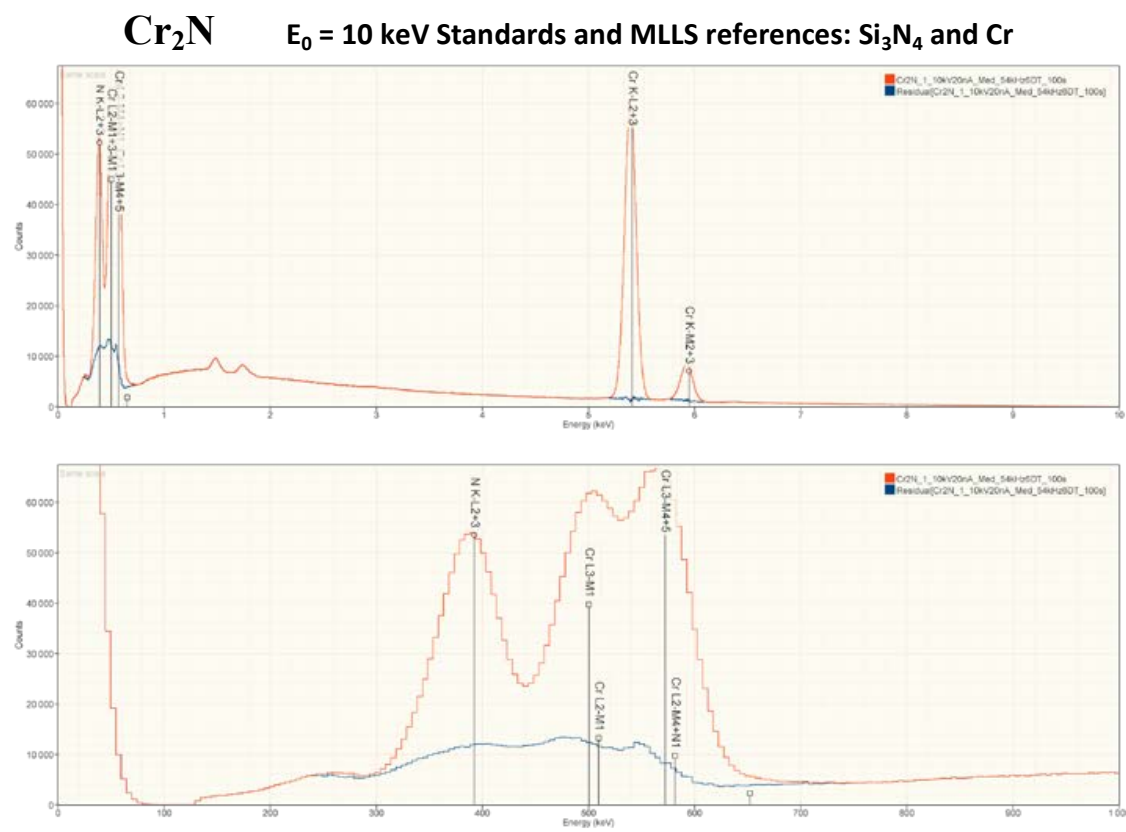

Table 1 SEM/SDD-EDS analysis of metal carbides (results in atom fractions); 5 replicates

\begin{tabular}{|l|l|l|l|l|l|l|l|l|l|l|}
\hline Carbide & C ideal & $\begin{array}{l}\text { C } \\
\text { mean }\end{array}$ & $\begin{array}{l}\text { Relative } \\
\text { error, } \%\end{array}$ & $\sigma$ & $\begin{array}{l}\text { Relative } \\
\sigma, \%\end{array}$ & $\begin{array}{l}\text { metal } \\
\text { ideal }\end{array}$ & $\begin{array}{l}\text { metal } \\
\text { mean }\end{array}$ & $\begin{array}{l}\text { Relative } \\
\text { error, \% }\end{array}$ & $\begin{array}{l}\sigma \\
\text { Relative } \\
\sigma, \%\end{array}$ \\
\hline CГ3C2 & $\mathbf{0 . 4 0 0 0}$ & $\mathbf{0 . 3 9 6 7}$ & $\mathbf{- 0 . 8 3 \%}$ & $\mathbf{0 . 0 0 2 9}$ & $\mathbf{0 . 7 3 \%}$ & $\mathbf{0 . 6 0 0 0}$ & $\mathbf{0 . 6 0 3 3}$ & $\mathbf{0 . 5 5 \%}$ & $\mathbf{0 . 0 0 2 9}$ & $\mathbf{0 . 4 8 \%}$ \\
\hline $\mathbf{C}_{7} \mathbf{C}_{3}$ & $\mathbf{0 . 3 0 0 0}$ & $\mathbf{0 . 2 9 6 1}$ & $\mathbf{- 1 . 3 \%}$ & $\mathbf{0 . 0 0 9 8}$ & $\mathbf{3 . 3 \%}$ & $\mathbf{0 . 7 0 0 0}$ & $\mathbf{0 . 7 0 3 9}$ & $\mathbf{0 . 5 7 \%}$ & $\mathbf{0 . 0 0 9 8}$ & $\mathbf{1 . 4 \%}$ \\
\hline${ }^{{ }_{2} 23} \mathbf{C}_{6}$ & $\mathbf{0 . 2 0 6 9}$ & $\mathbf{0 . 2 0 6 9}$ & $\mathbf{0 \%}$ & $\mathbf{0 . 0 0 3 9}$ & $\mathbf{1 . 9} \%$ & $\mathbf{0 . 7 9 3 1}$ & $\mathbf{0 . 7 9 3 1}$ & $\mathbf{0} \%$ & $\mathbf{0 . 0 0 3 9}$ & $\mathbf{0 . 4 9 \%}$ \\
\hline Fe3C & $\mathbf{0 . 2 5 0 0}$ & $\mathbf{0 . 2 4 6 9}$ & $\mathbf{- 1 . 2 \%}$ & $\mathbf{0 . 0 0 0 8 5}$ & $\mathbf{0 . 3 5 \%}$ & $\mathbf{0 . 7 5 0 0}$ & $\mathbf{0 . 7 5 3 1}$ & $\mathbf{0 . 4 2 \%}$ & $\mathbf{0 . 0 0 0 8 5}$ & $\mathbf{0 . 1 1 \%}$ \\
\hline
\end{tabular}

Table 2 SEM/SDD-EDS analysis of metal nitrides (results in atom fractions); 5 replicates

\begin{tabular}{|l|l|l|l|l|l|l|l|l|l|l|}
\hline Nitride & N ideal & $\begin{array}{l}\mathrm{N} \\
\text { mean }\end{array}$ & $\begin{array}{l}\text { Relative } \\
\text { error, } \%\end{array}$ & $\sigma$ & $\begin{array}{l}\text { Relative } \\
\sigma, \%\end{array}$ & $\begin{array}{l}\text { metal } \\
\text { ideal }\end{array}$ & $\begin{array}{l}\text { metal } \\
\text { mean }\end{array}$ & $\begin{array}{l}\text { Relative } \\
\text { error, \% }\end{array}$ & $\sigma$ & $\begin{array}{l}\text { Relative } \\
\sigma, \%\end{array}$ \\
\hline TiN & $\mathbf{0 . 5 0 0 0}$ & $\mathbf{0 . 5 0 9 8}$ & $\mathbf{2 . 0 \%}$ & $\mathbf{0 . 0 0 1 5 6}$ & $\mathbf{0 . 3 \%}$ & $\mathbf{0 . 5 0 0 0}$ & $\mathbf{0 . 4 9 0 2}$ & $\mathbf{- 2 . 0 \%}$ & $\mathbf{0 . 0 0 1 5 6}$ & $\mathbf{0 . 3 2 \%}$ \\
\hline VN & $\mathbf{0 . 3 0 0 0}$ & $\mathbf{0 . 2 9 6 1}$ & $\mathbf{- 1 . 3 \%}$ & $\mathbf{0 . 0 0 9 8}$ & $\mathbf{3 . 3} \%$ & $\mathbf{0 . 7 0 0 0}$ & $\mathbf{0 . 7 0 3 9}$ & $\mathbf{0 . 5 7 \%}$ & $\mathbf{0 . 0 0 9 8}$ & $\mathbf{1 . 4 \%}$ \\
\hline Cr2N & $\mathbf{0 . 3 3 3 3}$ & $\mathbf{0 . 3 5 0 1}$ & $\mathbf{5 . 1 \%}$ & $\mathbf{0 . 0 0 6}$ & $\mathbf{1 . 7} \%$ & $\mathbf{0 . 6 6 6 7}$ & $\mathbf{0 . 6 4 9 9}$ & $\mathbf{- 2 . 5 \%}$ & $\mathbf{0 . 0 0 6}$ & $\mathbf{0 . 9 3 \%}$ \\
\hline Fe3N & $\mathbf{0 . 2 5 0 0}$ & $\mathbf{0 . 2 5 7 3}$ & $\mathbf{2 . 9 \%}$ & $\mathbf{0 . 0 0 6 8 8}$ & $\mathbf{2 . 7} \%$ & $\mathbf{0 . 7 5 0 0}$ & $\mathbf{0 . 7 4 2 7}$ & $\mathbf{- 1 . 0 \%}$ & $\mathbf{0 . 0 0 6 8 8}$ & $\mathbf{0 . 9 0 \%}$ \\
\hline
\end{tabular}

Table 3 SEM/SDD-EDS analysis of metal oxides (results in atom fractions); 5 replicates

\begin{tabular}{|l|l|l|l|l|l|l|l|l|l|l|}
\hline Oxide & O ideal & $\begin{array}{l}\text { O } \\
\text { mean }\end{array}$ & $\begin{array}{l}\text { Relative } \\
\text { error, } \%\end{array}$ & $\sigma$ & $\begin{array}{l}\text { Relative } \\
\sigma, \%\end{array}$ & $\begin{array}{l}\text { metal } \\
\text { ideal }\end{array}$ & $\begin{array}{l}\text { metal } \\
\text { mean }\end{array}$ & $\begin{array}{l}\text { Relative } \\
\text { error, \% }\end{array}$ & $\begin{array}{l}\sigma \\
\text { Relative } \\
\sigma, \%\end{array}$ \\
\hline AI2O3 & $\mathbf{0 . 6 0 0 0}$ & $\mathbf{0 . 5 9 0 5}$ & $-\mathbf{- 1 . 6 \%}$ & $\mathbf{0 . 0 0 0 3 9}$ & $\mathbf{0 . 0 7 \%}$ & $\mathbf{0 . 4 0 0 0}$ & $\mathbf{0 . 4 0 9 5}$ & $\mathbf{2 . 4 \%}$ & $\mathbf{0 . 0 0 0 3 9}$ & $\mathbf{0 . 1 \%}$ \\
\hline SÍO2 & $\mathbf{0 . 6 6 6 7}$ & $\mathbf{0 . 6 5 3 5}$ & $\mathbf{- 2 . 0 \%}$ & $\mathbf{0 . 0 0 0 3 3}$ & $\mathbf{0 . 0 5 \%}$ & $\mathbf{0 . 3 3 3 3}$ & $\mathbf{0 . 3 4 6 5}$ & $\mathbf{4 . 0} \%$ & $\mathbf{0 . 0 0 0 3 3}$ & $\mathbf{0 . 1 \%}$ \\
\hline SiO & $\mathbf{0 . 5 0 0 0}$ & $\mathbf{0 . 4 9 8 9}$ & $\mathbf{- 0 . 2 0} \%$ & $\mathbf{0 . 0 0 0 3 3}$ & $\mathbf{0 . 0 7 \%}$ & $\mathbf{0 . 5 0 0 0}$ & $\mathbf{0 . 5 0 1 1}$ & $\mathbf{0 . 2 0} \%$ & $\mathbf{0 . 0 0 0 3 3}$ & $\mathbf{0 . 0 7 \%}$ \\
\hline Fe2O3 & $\mathbf{0 . 6 0 0 0}$ & $\mathbf{0 . 5 9 8 8}$ & $\mathbf{- 0 . 2 0} \%$ & $\mathbf{0 . 0 0 0 6 8}$ & $\mathbf{0 . 1 1 \%}$ & $\mathbf{0 . 4 0 0 0}$ & $\mathbf{0 . 4 0 1 2}$ & $\mathbf{0 . 3 0 \%}$ & $\mathbf{0 . 0 0 0 6 8}$ & $\mathbf{0 . 1 7 \%}$ \\
\hline CU2O & $\mathbf{0 . 3 3 3 3}$ & $\mathbf{0 . 3 2 5 0}$ & $\mathbf{- 2 . 5 \%}$ & $\mathbf{0 . 0 0 1 0}$ & $\mathbf{0 . 3 0} \%$ & $\mathbf{0 . 6 6 6 7}$ & $\mathbf{0 . 6 7 5 0}$ & $\mathbf{1 . 2 \%}$ & $\mathbf{0 . 0 0 1 0}$ & $\mathbf{0 . 1 5 \%}$ \\
\hline CuO & $\mathbf{0 . 5 0 0 0}$ & $\mathbf{0 . 4 8 6 8}$ & $\mathbf{- 2 . 6 \%}$ & $\mathbf{0 . 0 0 2 6}$ & $\mathbf{0 . 5 4 \%}$ & $\mathbf{0 . 5 0 0 0}$ & $\mathbf{0 . 5 1 3 2}$ & $\mathbf{2 . 6 \%}$ & $\mathbf{0 . 0 0 2 6}$ & $\mathbf{0 . 5 1 \%}$ \\
\hline
\end{tabular}

